\title{
Evolution of dust grain size distribution by shattering in the interstellar medium: Robustness and uncertainty
}

\author{
Hiroyuki Hirashita $^{1}$ and Hiroshi Kobayashi ${ }^{2}$ \\ ${ }^{1}$ Institute of Astronomy and Astrophysics, Academia Sinica, P.O. Box 23-141, Taipei 10617, Taiwan \\ ${ }^{2}$ Department of Physics, Nagoya University, Nagoya, Aichi 464-8602, Japan
}

(Received November 16, 2012; Revised February 27, 2013; Accepted March 9, 2013; Online published October 24, 2013)

\begin{abstract}
Shattering of dust grains in the interstellar medium is a viable mechanism of small grain production in galaxies. We examine the robustness or uncertainty in the theoretical predictions of shattering. We identify $P_{1}$ (the critical pressure above which the deformation destroys the original lattice structures) as the most important quantity in determining the timescale of small grain production, and confirm that the same $P_{1} / t$ ( $t$ is the duration of shattering) gives the same grain size distribution $[n(a)$, where $a$ is the grain radius] after shattering within a factor of 3. The uncertainty in the fraction of shocked material that is eventually ejected as fragments causes uncertainties in $n(a)$ by a factor of 1.3 and 1.6 for silicate and carbonaceous dust, respectively. The size distribution of shattered fragments have minor effects as long as $\alpha_{\mathrm{f}} \lesssim 3.5$ (the size distribution of shattered fragments $\left.\propto a^{-\alpha_{\mathrm{f}}}\right)$, since the slope of grain size distribution $n(a)$ continuously changes by shattering and becomes consistent with $n(a) \propto a^{-3.5}$. The grain velocities as a function of grain radius can have an imprint in the grain size distribution especially for carbonaceous dust. We also show that the formulation of shattering can be simplified without losing sufficient precision.
\end{abstract}

Key words: Cosmic dust, galaxy evolution, grain size distribution, interstellar medium.

\section{Introduction}

The evolution of dust in galaxies is important to the understanding of galaxy evolution, since dust grains govern some fundamental physical processes in the interstellar medium (ISM). First, they dominate the absorption and scattering of the stellar light, affecting the radiative transfer in the ISM. Second, the dust surface is the main site for the formation of molecular hydrogen. The former process is governed by the extinction curve (absorption and scattering coefficient as a function of wavelength; Hoyle and Wickramasinghe, 1969; Draine, 2003) and the latter by the total surface area of dust grains (Yamasawa et al., 2011). Since the extinction curve and the total grain surface area both depend strongly on the grain size distribution, clarifying the regulating mechanism of grain size distribution is of particular importance in understanding those important roles of dust.

Mathis et al. (1977, hereafter MRN) show that a mixture of silicate and graphite with a grain size distribution [number density of grains per grain radius, denoted as $n(a)$ in this paper] proportional to $a^{-3.5}$ (a grain size distribution with a power index of -3.5 is called MRN grain size distribution), where $a$ is the grain radius ( $a \sim 0.001-0.25 \mu \mathrm{m}$ ), reproduces the Milky Way extinction curve. Pei (1992) shows that the extinction curves in the Magellanic Clouds are also explained by the MRN grain size distribution with different

Copyright (C) The Society of Geomagnetism and Earth, Planetary and Space Sciences (SGEPSS); The Seismological Society of Japan; The Volcanological Society of Japan; The Geodetic Society of Japan; The Japanese Society for Planetary Sciences; TERRAPUB.

doi:10.5047/eps.2013.03.008 abundance ratios between silicate and graphite. Kim et al. (1994) and Weingartner and Draine (2001) have applied a more detailed fit to the Milky Way extinction curve in order to obtain the grain size distribution. Although their grain size distributions deviate from the MRN size distribution, the overall trend from small to large grain sizes roughly follows a power law with an index near to -3.5 . In any models that fit the Milky Way extinction curve, the existence of a large number of small $(a \lesssim 0.01 \mu \mathrm{m})$ grains is required. The existence of such small grains is further supported by the mid-infrared excess of the spectral energy distribution in the Milky Way (e.g., Désert et al., 1990; Draine and Li, 2001).

Dust grains formed by stellar sources, mainly supernovae (SNe) and asymptotic giant branch (AGB) stars, are not sufficient to explain the total dust mass in the Milky Way ISM, because the timescale of dust enrichment by these sources is much longer than that of dust destruction by SN shocks (e.g., McKee, 1989; Draine, 1995). Moreover, both SNe and AGB stars are suggested to supply large $(a \gtrsim$ $0.1 \mu \mathrm{m})$ grains into the ISM and cannot be the dominant source of small grains (Nozawa et al., 2007; Höfner, 2008; Mattsson and Höfner, 2011; Norris et al., 2012). Thus, some interstellar processes (or more precisely, non-stellar processes) are necessary to explain the large abundance of small grains.

There are some possible processes that efficiently modify the grain size distribution. Hellyer (1970) shows that the collisional fragmentation of dust grains finally leads to a power-law grain size distribution similar to the MRN size distribution (see also Bishop and Searle, 1983; Tanaka 
et al., 1996). Hirashita and Yan (2009) show that such a fragmentation and disruption process (or shattering) can be driven efficiently by turbulence in the diffuse ISM. Dust grains are also processed by other mechanisms. Various authors show that the increase of the total dust mass in the Milky Way ISM is mainly governed by grain growth through the accretion of gas phase metals onto the grains (we call elements composing dust grains "metals") (e.g., Dwek, 1998; Zhukovska et al., 2008; Inoue, 2011; Asano et al., 2013a). Grain growth mainly works in the dense ISM, such as molecular clouds. In the dense ISM, coagulation also occurs, making the grain sizes larger (e.g., Hirashita and Yan, 2009; Ormel et al., 2009). In the diffuse ISM phase, interstellar shocks associated with supernova (SN) remnants destroy dust grains, especially small ones, by sputtering (e.g., McKee, 1989; Nozawa et al., 2006). Shattering also occurs in SN shocks (Jones et al., 1996). All these processes above other than shattering do not contribute efficiently to the increase of small $(a \lesssim 0.01 \mu \mathrm{m})$ grain abundance. Indeed, Asano et al. (2013b) show that, unless we consider shattering, the grain size distribution is biased to large ( $a \sim 0.1 \mu \mathrm{m})$ sizes. Therefore, shattering is important in reprocessing large grains into small grains.

Based on the formulation developed by Hirashita and Yan (2009), Hirashita et al. (2010, hereafter H10) consider small grain production by shattering in the context of galaxy evolution. H10 assume that Type II SNe (SNe II) are the source of the first dust grains, which are biased to large sizes $(\gtrsim 0.1 \mu \mathrm{m})$ because small grains are destroyed in the shocked region in the SNe II (Nozawa et al., 2007, hereafter N07). Starting with the size distribution of SNe II dust grains in N07, H10 solve the shattering equation to calculate the evolution of grain size distribution. They show that the velocity dispersions acquired by the dust grains in a warm ionized medium (WIM) is large enough for shattering to produce a large abundance of small grains on a short $(\lesssim 10$ Myr) timescale. As mentioned above, even if we consider other sources of dust such as the dust formation in the wind of AGB stars and the accretion of metals onto grains in the interstellar clouds, shattering is generally necessary to produce small grains (Asano et al., 2013b).

Considering that shattering is a unique mechanism that produces small grains efficiently in the ISM, it is important to clarify how robust or uncertain the theoretical calculations of shattering are. Since there are some basic physical parameters regulating shattering, it is crucial to clarify how they affect the grain size distribution or to which parameter shattering is sensitive. Although any models should commonly contain those basic parameters, there could be some uncertainties in the formulation itself, or there could be unnecessary complexity which cannot be constrained from observations anyway. Thus, in this paper, we examine the robustness of shattering calculations for the interstellar dust by changing some major parameters in a model and comparing the results between two different frameworks.

This paper is organized as follows. In Section 2, we explain the shattering models and pick out some major parameters. In Section 3, we examine the variation of the grain size distribution due to changes of the major parameters. In Section 4 , we discuss our results and implications for the evolution of grain size distribution. In Section 5, we give our conclusions.

\section{Overview of the Models for Shattering}

As mentioned in Introduction, we use the framework in $\mathrm{H} 10$ to test the robustness of a shattering model. H10 consider the evolution of grain size distribution through shattering by solving a shattering equation, which treats the grain-grain collision rate and the redistribution of shattered fragments in the grain size distribution. They assume that grains collide with each other under the velocity dispersions induced by dynamical coupling with interstellar turbulence. The production of fragments in grain-grain collisions is the key process that we focus on below.

The dust grains are assumed to be spherical with radius $a$. Thus, the grain mass $m$ is related to the grain radius by

$$
m=\frac{4}{3} \pi a^{3} \rho_{\mathrm{gr}},
$$

where $\rho_{\mathrm{gr}}$ is the material density of the grain. The grain size distribution, $n(a)$, is defined so that $n(a) d a$ is the number density of grains with radii between $a$ and $a+d a$.

\subsection{Initial grain size distribution}

The size distribution of grains ejected from SNe II into the ISM is adopted from N07. This size distribution is used as the initial condition for the calculation of shattering. N07 treated dust nucleation and growth in a SN II, taking into account the dust destruction by kinetic and thermal sputtering in the shocked region. Thus, the grain size distribution calculated by N07 is regarded as that ejected from SNe II to the ISM (see also Bianchi and Schneider, 2007). Following H10, we adopt $20 \mathrm{M}_{\odot}$ as a representative progenitor mass, and the unmixed case in which the original onion-like structure of elements is preserved, since the extinction features of carbon and silicon, which are major grain components in the unmixed case, are consistent with observations (Hirashita et al., 2005; Kawara et al., 2011). The formed grain species are $\mathrm{C}, \mathrm{Si}, \mathrm{SiO}_{2}, \mathrm{Fe}, \mathrm{FeS}, \mathrm{Al}_{2} \mathrm{O}_{3}$, $\mathrm{MgO}, \mathrm{MgSiO}_{3}$, and $\mathrm{Mg}_{2} \mathrm{SiO}_{4}$. According to N07, small grains with $a \lesssim 0.02 \mu \mathrm{m}$ are trapped in the shocked region and are efficiently destroyed by thermal sputtering if the ambient hydrogen number density, $n_{\mathrm{H}}$, is larger than 0.1 $\mathrm{cm}^{-3}$. In this paper, we adopt $n_{\mathrm{H}}=1 \mathrm{~cm}^{-3}$ (Subsection 2.2). As shown in N07 (see also H10), most of the grains have radii $0.1-1 \mu \mathrm{m}$. The initial grain size distributions are also shown later in Fig. 2.

The normalization of the grain size distribution is determined as follows. We consider a solar metallicity environment as a representative case. (For other metallicities, the timescale of shattering just scales with the inverse of metallicity.) We parameterize the dust abundance by the oxygen abundance. The oxygen mass produced by a SN II of $20 \mathrm{M}_{\odot}$ progenitor is $m_{\mathrm{O}}=1.58 \mathrm{M}_{\odot}$ according to Umeda and Nomoto (2002), whose data were adopted by N07. The solar oxygen abundance is assumed to be $Z_{\mathrm{O}, \odot}=5.6 \times 10^{-3}$ by mass ratio (Lodders, 2003). Thus, for the solar oxygen abundance, the corresponding dust-to-gas ratio is given by $\mathcal{D}_{0}=Z_{\mathrm{O}, \odot} m_{\mathrm{d}} / m_{\mathrm{O}}$, where $m_{\mathrm{d}}$ is the dust mass ejected from a single SN II $\left(0.14 \mathrm{M}_{\odot}\right.$; N07; H10). Here we assume that both oxygen and dust are supplied from SNe II. The grain 
Table 1. Summary of experimental properties.

\begin{tabular}{lccccc}
\hline Species & $\begin{array}{c}\rho_{\mathrm{gr}} \\
\left(\mathrm{g} \mathrm{cm}^{-3}\right)\end{array}$ & $\begin{array}{c}c_{0} \\
\left(\mathrm{~km} \mathrm{~s}^{-1}\right)\end{array}$ & $s$ & $\begin{array}{c}v_{\text {shat }} \\
\left(\mathrm{km} \mathrm{s}^{-1}\right)\end{array}$ & $\begin{array}{c}P_{1}{ }^{\mathrm{a}} \\
\left(\mathrm{dyn} \mathrm{cm}^{-2}\right)\end{array}$ \\
\hline Silicate & 3.3 & 5 & 1.2 & 2.7 & $3 \times 10^{11}$ \\
Carbonaceous dust & 2.2 & 1.8 & 1.9 & 1.2 & $4 \times 10^{10}$ \\
\hline
\end{tabular}

${ }^{\text {a }} P_{1}$ is varied in Models $\mathrm{D}$ and $\mathrm{E}$ in Table 2.

Note: All the quantities are determined experimentally. See Jones et al. (1996) and references therein.

size distribution is normalized so that the total grain mass density integrated for all the size range, $\rho_{\text {dust }}$, is equal to $1.4 n_{\mathrm{H}} m_{\mathrm{H}} \mathcal{D}_{0}$, where $m_{\mathrm{H}}$ is the mass of hydrogen atom, and the factor 1.4 is the correction for the species other than hydrogen.

As mentioned in Introduction, additional contribution from AGB stars does not change the following results significantly as long as AGB stars also supply large $(a \gtrsim$ $0.1 \mu \mathrm{m}$ ) grains (see also Asano et al., 2013b). In other words, the use of N07's results for the initial condition is aimed at investigating the important role of shattering in the production of small grains from large grains.

\subsection{Shattering}

We calculate the shattering processes in the same way as in $\mathrm{H} 10$ by solving the shattering equation. The collision frequency between grains with various sizes is determined by grain-size-dependent velocity dispersions. The shattering equation used in H10 is based on Hirashita and Yan (2009) (originally taken from Jones et al., 1994, 1996). Shattering is assumed to take place if the relative velocity between a pair of grains is larger than the shattering threshold velocities, $v_{\text {shat }}\left(2.7\right.$ and $1.2 \mathrm{~km} \mathrm{~s}^{-1}$ for silicate and graphite, respectively; Jones et al., 1996). The results are not sensitive to $v_{\text {shat }}$ as long as the grain velocities driven by turbulence is much larger than the threshold, but are rather sensitive to the hardness of the grain materials as shown later.

We consider a range $a_{\min }=3 \times 10^{-8} \mathrm{~cm}(3 \AA)$ and $a_{\max }=3 \times 10^{-4} \mathrm{~cm}(3 \mu \mathrm{m})$ for the grain radii. The grains passing through the boundary of the smallest radius are removed from the calculation. Most grain models adopt a minimum grain radius of a few $\times 10^{-8} \mathrm{~cm}$ (Weingartner and Draine, 2001; Guillet et al., 2009) although applying bulk material properties to such small grains could cause a large uncertainty. However, even if we adopt $a_{\min }=$ $10^{-7} \mathrm{~cm}$, the results does not change, except that the grain size distribution is truncated at $10^{-7} \mathrm{~cm}$. This is because shattering occurs in a top-down manner in the grain sizes and the production of grains with $a \sim$ a few $\times 10^{-8} \mathrm{~cm}$ by shattering is never enough for these small grains to have a large contribution to the total shattering rate.

Although nine grain species are predicted to form (Subsection 2.1), the material properties needed for the calculation of shattering are not necessarily available for all the species. Thus, we divide the grains into two groups: one is carbonaceous dust and the other is all the other species of dust (called "silicate"), and apply the relevant material quantities of graphite and silicate, respectively. The material properties of silicate and graphite are taken from Jones et al. (1996) and summarized in Table 1.

Since the shattering equation is general enough, the major uncertainties can be produced by the treatment of shat- tering fragments. Thus, we explain how to treat shattering fragments below.

We consider two colliding grains whose masses are $m_{1}$ and $m_{2}$ (the former grain is called target grain), and estimate the total fragment mass in $m_{1}$ as a result of this collision. Note that we consider a collision between $m_{2}$ and $m_{1}$ again in the calculation and consider the fragments in $m_{2}$ (that is, we consider the same collision twice to treat the fragments of each colliding grain). The total fragment mass is determined by the mass shocked to the critical pressure in the target (Jones et al., 1996; Hirashita and Yan, 2009):

$$
\frac{M_{\mathrm{ej}}}{m_{2}}=f_{M} \frac{M}{m_{2}}=\frac{f_{M}(1+2 \mathcal{R})}{2(1+\mathcal{R})^{9 / 16}} \frac{1}{\sigma_{r}^{1 / 9}}\left(\frac{\mathcal{M}_{r}^{2}}{\sigma_{1} \mathcal{M}_{1}^{2}}\right)^{8 / 9},
$$

where $M_{\text {ej }}$ is the total mass of fragments ejected from $m_{1}$, $M$ is the mass shocked to the critical pressure $\left(P_{1}\right)$, above which the solid becomes plastic (that is, the deformation destroys the original lattice structures), $f_{M}$ is the fraction of shocked mass that is eventually ejected as fragments (the rest remains in the grain), $\mathcal{R}=1$ in the collision between the same species (we only consider collisions between the same species for simplicity), $\mathcal{M}_{r} \equiv v / c_{0}\left(c_{0}\right.$ is the sound speed of the grain material), $\mathcal{M}_{1}$ is the Mach number corresponding to the critical pressure $P_{1}$ :

$$
\mathcal{M}_{1}=\frac{2 \phi_{1}}{1+\left(1+4 s \phi_{1}\right)^{1 / 2}}
$$

where $\phi_{1} \equiv P_{1} /\left(\rho_{\mathrm{gr}} c_{0}^{2}\right)$ and $s$ is a dimensionless material constant that determines the relation between the shocked velocity and the velocity of the shocked matter. For convenience, we define function $\sigma$ as

$$
\sigma(\mathcal{M}) \equiv \frac{0.30\left(s+\mathcal{M}^{-1}-0.11\right)^{1.3}}{s+\mathcal{M}^{-1}-1},
$$

and evaluate $\sigma_{1}=\sigma\left(\mathcal{M}_{1}\right)$ and $\sigma_{r}=\sigma\left(\mathcal{M}_{r} /(1+\mathcal{R})\right)$ in Eq. (2). If $M$ is larger than half of the grain mass, we assume that the whole grain is fragmented; i.e., $M_{\mathrm{ej}}=m_{1}$. This case is called catastrophic disruption. Otherwise (i.e., for $\left.M<m_{1} / 2\right)$, only a fraction of $m_{1}\left(f_{M} M\right)$ is ejected as fragments. This case is called cratering. The relation between the total volume $(V)$ of shattered fragments and the critical pressure is roughly given by the total energy $P_{1} V=$ constant. Indeed, the above equations tell us that $M \propto V$ is inversely proportional to $P_{1}$, if $4 s \phi_{1}>1$, which is true for most of the cases considered in this paper $\left(\mathcal{M}_{1}^{2} \sim \phi_{1}\right.$, so $\left.M_{\mathrm{ej}} \propto \phi_{1}^{-8 / 9} \propto P_{1}^{-8 / 9}\right)$. Since the shattering efficiency is proportional to $M_{\mathrm{ej}}$, the timescale of shattering is regulated by $P_{1}$. Considering that the material properties of astronomical grains are uncertain, we vary $P_{1}$ (Subsection 2.4.2). 
Table 2. Models.

\begin{tabular}{|c|c|c|c|c|c|}
\hline Name & Species & $f_{M}$ & 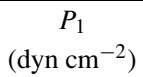 & $\alpha_{\mathrm{f}}$ & Grain velocities \\
\hline \multirow[t]{2}{*}{$A^{a}$} & silicate & 0.4 & $3 \times 10^{11}$ & 3.3 & Yan et al. (2004) \\
\hline & graphite & 0.4 & $4 \times 10^{10}$ & 3.3 & Yan et al. (2004) \\
\hline \multirow[t]{2}{*}{ B } & silicate & 0.3 & $3 \times 10^{11}$ & 3.3 & Yan et al. (2004) \\
\hline & graphite & 0.3 & $4 \times 10^{10}$ & 3.3 & Yan et al. (2004) \\
\hline \multirow[t]{2}{*}{$\mathrm{C}$} & silicate & 0.6 & $3 \times 10^{11}$ & 3.3 & Yan et al. (2004) \\
\hline & graphite & 0.6 & $4 \times 10^{10}$ & 3.3 & Yan et al. (2004) \\
\hline \multirow[t]{2}{*}{$\mathrm{D}^{\mathrm{a}}$} & silicate & 0.4 & $1 \times 10^{11}$ & 3.3 & Yan et al. (2004) \\
\hline & graphite & 0.4 & $1.3 \times 10^{10}$ & 3.3 & Yan et al. (2004) \\
\hline \multirow[t]{2}{*}{$\mathrm{E}^{\mathrm{a}}$} & silicate & 0.4 & $9 \times 10^{11}$ & 3.3 & Yan et al. (2004) \\
\hline & graphite & 0.4 & $1.2 \times 10^{11}$ & 3.3 & Yan et al. (2004) \\
\hline \multirow[t]{2}{*}{$\mathrm{F}$} & silicate & 0.4 & $3 \times 10^{11}$ & 2.3 & Yan et al. (2004) \\
\hline & graphite & 0.4 & $4 \times 10^{10}$ & 2.3 & Yan et al. (2004) \\
\hline \multirow[t]{2}{*}{ G } & silicate & 0.4 & $3 \times 10^{11}$ & 4.3 & Yan et al. (2004) \\
\hline & graphite & 0.4 & $4 \times 10^{10}$ & 4.3 & Yan et al. (2004) \\
\hline \multirow[t]{2}{*}{$\mathrm{H}$} & silicate & 0.4 & $3 \times 10^{11}$ & 3.3 & Eq. (10) \\
\hline & graphite & 0.4 & $4 \times 10^{10}$ & 3.3 & Eq. (10) \\
\hline
\end{tabular}

a'We also examine KT10's formulation for fragments (Section 2.4.2).

The total fragment mass $M_{\mathrm{ej}}$ is distributed with a grain size distribution $\propto a^{-\alpha_{\mathrm{f}}}$. Jones et al. (1996) argue that $\alpha_{\mathrm{f}}=3.0-3.4$, based on an analysis of the flow associated with the formation of a crater (see also Takagi et al., 1984; Nakamura and Fujiwara, 1991; Nakamura et al., 1994; Takasawa et al., 2011 for experimental results). Unless the catastrophic disruption occurs, we assume that the mass $m_{1}-M_{\mathrm{ej}}$ remains as a single dust grain and is distributed in an appropriate bin in the numerical calculation. To avoid unnecessary complexity caused by the choice of the upper and lower radii of the fragments, we adopt simpler forms for the smallest and largest fragments than H11, following Guillet et al. (2009):

$$
a_{f \max }=(0.0204 f)^{1 / 3} a_{1},
$$

where $a_{1}$ is the radius of the grain $m_{1}$ (i.e., the target grain), $f \equiv M_{\mathrm{ej}} / m_{1}$ (shattered fraction of the target grain). The minimum radius is assumed to be $a_{f, \min }=a_{\min }=3 \times$ $10^{-8} \mathrm{~cm}$. If $a_{f, \max }<a_{\min }$, we remove the fragments from the calculation.

The relative velocity in the collision is estimated based on the grain velocity dispersion as a function of grain radius $a$. We used the calculation by Yan et al. (2004), who consider the grain acceleration by hydrodrag and gyroresonance in magnetohydrodynamic turbulence, and calculate the grain velocities achieved in various phases of ISM. Among the ISM phases, we focus on the warm ionized medium (WIM) to investigate the possibility of efficient shattering in actively star-forming environments. We adopt $n_{\mathrm{H}}=1 \mathrm{~cm}^{-3}$ for the hydrogen number density of the WIM. The resulting grain size distribution is not sensitive to $n_{\mathrm{H}}(\mathrm{H} 10)$. Indeed, if $n_{\mathrm{H}}$ is large, the grain-grain collision rate (i.e., the shattering efficiency) rises, while dust grains are more destroyed in $\mathrm{SNe}$ (i.e., the dust-to-gas ratio is lower). Because of these compensating effects, the resulting grain size distribution is not sensitive to the gas density. We adopt gas temperature $T=8000 \mathrm{~K}$, electron number density $n_{\mathrm{e}}=n_{\mathrm{H}}$, Alfvén speed $V_{\mathrm{A}}=20 \mathrm{~km} \mathrm{~s}^{-1}$ and injection scale of the turbulence
$L=100 \mathrm{pc}$. For grains with $a \gtrsim 0.1 \mu \mathrm{m}$, where most of the grain mass is contained in our cases, the grain velocity is governed by gyroresonance. Since there is still an uncertainty in the typical grain radius $\left(a_{\mathrm{c}}\right)$ above which gyroresonance effectively works, we also address the variation of results by $a_{\mathrm{c}}$ (Subsection 2.4.4).

The grain velocities given above are velocity dispersions. In order to estimate the relative velocity $\left(v_{12}\right)$ between the grains with $m_{1}$ and $m_{2}$, whose velocity dispersions are $v_{1}$ and $v_{2}$, respectively, and we average the size distributions of fragments for four cases $v_{12}=v_{1}+v_{2},\left|v_{1}-v_{2}\right|, v_{1}$, and $v_{2}$ to take the directional variety into account. See $\mathrm{H} 10$ for more details.

\subsection{Duration of shattering}

Since we consider shattering in the WIM, it is reasonable to assume that the shattering duration (denoted as $t$ ) is determined by a timescale on which the ionization is maintained. A typical lifetime of ionizing stars is $\lesssim 10 \mathrm{Myr}$ (Bressan et al., 1993; Inoue et al., 2000). We basically adopt $t=10$ Myr in this paper, but we also consider longer durations for a longer starburst duration or shattering over multiple starburst episodes.

\subsection{Parameters}

Based on the discussions in the previous subsection, we change the following parameters related to the fragments. The models are summarized in Table 2, where Model A adopts "fiducial" values for the parameters.

2.4.1 Ejected fraction of shocked material How much fraction of the shocked material is finally ejected as fragments is uncertain. This factor is denoted as $f_{M}$ in Eq. (2). According to Jones et al. (1996), $f_{M}$ is between 0.3 and 0.6 , while $\mathrm{H} 10$ adopted 0.4 . Thus, we adopt 0.4 as a fiducial value (Model A) and also examine 0.3 (Model B) and 0.6 (Model C).

2.4.2 Critical pressure Considering the uncertainty in the actual materials of astronomical grains, we vary $P_{1}$ by an order of magnitude as Models D and E in Table 2. The standard value adopted from Jones et al. (1996) is 

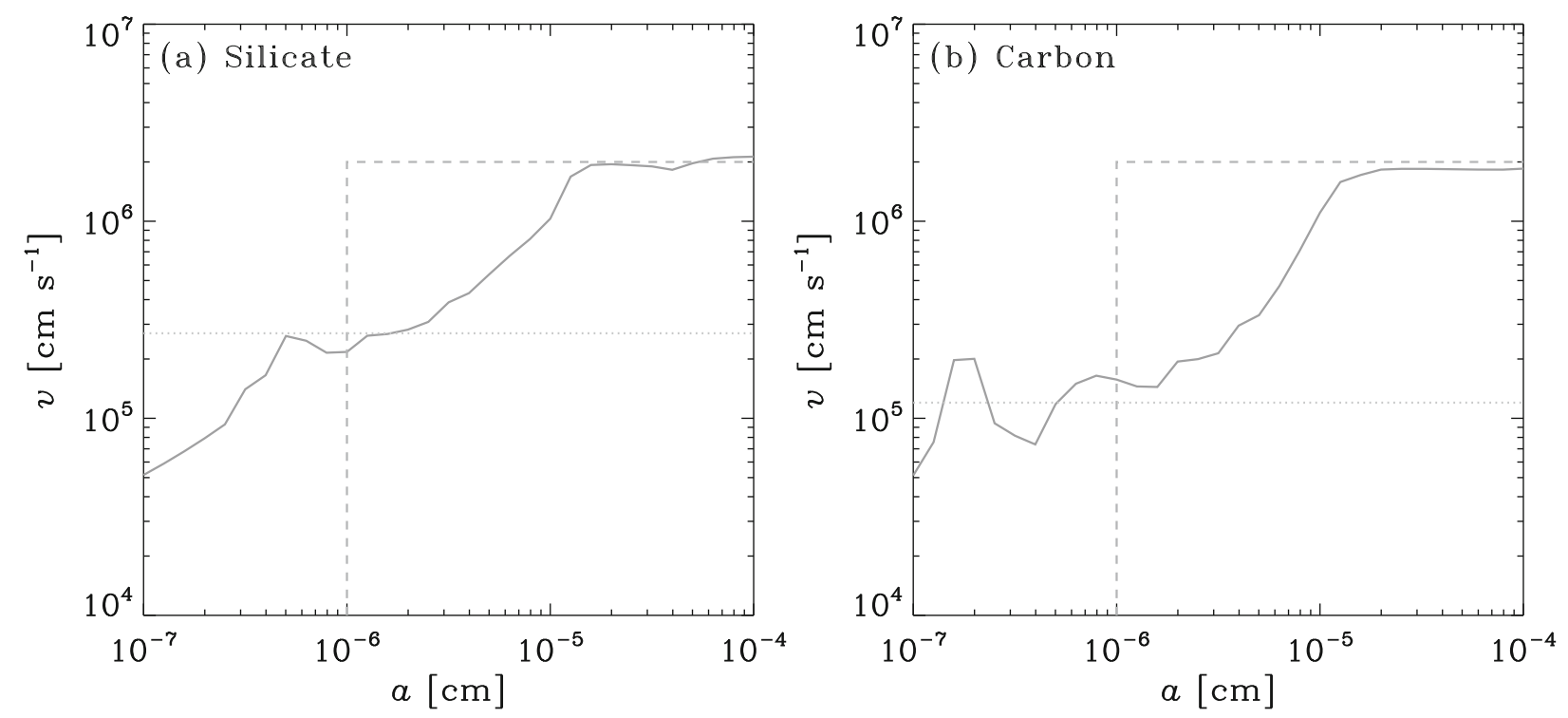

Fig. 1. Grain velocities $v$ as a function of grain radius $a$. Two grain species, (a) silicate and (b) carbonaceous dust, are shown. The solid lines indicate the velocity calculated by Yan et al. (2004) with the physical condition of the WIM described in the text. The dashed line shows the simplified velocity model (Eq. (10)) with $a_{\mathrm{c}}=10^{-6} \mathrm{~cm}$. The dotted line shows the shattering threshold velocity.

$P_{1}=3 \times 10^{11}$ and $4 \times 10^{10} \mathrm{dyn}^{-2}$ for silicate and graphite, respectively (Table 1; Model A in Table 2). We adopt three times smaller and larger values for $P_{1}$ in Models $\mathrm{D}$ and E, respectively. We call grains with low/high $P_{1}$ soft/hard grains.

There is another possible way of including the critical pressure in the model based on Kobayashi and Tanaka (2010, hereafter KT10). Jones et al. (1996) constructed the fragmentation model based on shock wave propagation caused by a collision (see also Mizutani et al., 1990). On the other hand, Holsapple (1987) provided a scaling formula for collisional outcomes based on laboratory experiments of his group (the review of the scaling formula is seen in Holsapple, 1993). In this formula, $M_{\mathrm{ej}}$ is proportional to

$$
\varphi=\frac{E_{\mathrm{imp}}}{m_{1} Q_{\mathrm{D}}^{\star}}
$$

for $\varphi \ll 1$, where

$$
E_{\mathrm{imp}}=\frac{1}{2} \frac{m_{1} m_{2}}{m_{1}+m_{2}} v^{2}
$$

is the impact energy between $m_{1}$ and $m_{2}$, and $Q_{\mathrm{D}}^{\star}$ is the specific impact energy at $M_{\mathrm{ej}}=m_{1} / 2$. Since $M_{\mathrm{ej}} \sim m_{1}$ for $\varphi \gg 1$, KT10 simply connected the cases for $\varphi \ll 1$ and $\varphi \gg 1$, providing a simple formula for $M_{\mathrm{ej}}$ as

$$
M_{\mathrm{ej}}=\frac{\varphi}{1+\varphi} m_{1} .
$$

Now we relate $Q_{\mathrm{D}}^{\star}$ to $P_{1}$. The dominant channel for the small grain production is cratering of large grains by small grains. If we assume that $m_{1} \gg m_{2}$ (and as long as $v^{2} / Q_{\mathrm{D}}^{\star}$ is not much larger than unity), we obtain $M_{\mathrm{ej}} \simeq$ $m_{2} v^{2} /\left(2 Q_{\mathrm{D}}^{\star}\right)$. On the other hand, if we approximate Eq. (2) as $M_{\mathrm{ej}} / m_{2} \simeq A\left(\rho_{\mathrm{gr}} v^{2} / P_{1}\right), A \sim 1$ for the range of quantities in this paper. Therefore, if the two models are equivalent, $Q_{\mathrm{D}}^{\star} \sim P_{1} /\left(2 \rho_{\mathrm{gr}}\right)$.
Thus, we examine KT10's formulation as follows. We adopt Eq. (8) instead of Eq. (2) for the total mass of fragments with the same fragment size distribution $\left(\propto a^{-\alpha_{\mathrm{f}}}\right.$ with the same upper and lower grain radii). The value of $\varphi$ is evaluated by Eqs. (6) and (7), where $Q_{\mathrm{D}}^{\star}$ is given by

$$
Q_{\mathrm{D}}^{\star}=\frac{P_{1}}{2 \rho_{\mathrm{gr}}} .
$$

This equation indicates that, if the energy per unit volume given to the grain exceeds $\sim P_{1} / \rho_{\mathrm{gr}}$, the major part of the grain is disrupted. We test Models A, D, and E by adopting KT10's formulation.

Note that we have adopted the approximation, $M_{\mathrm{ej}} \sim$ $\rho_{\mathrm{gr}} v^{2} / P_{1}$. This is a good approximation for $4 s \phi_{1}>1$ $\left(\phi_{1}=P_{1} /\left(\rho_{\mathrm{gr}} c_{0}^{2}\right)\right.$; see Subsection 2.2). However, if $\phi_{1} \ll$ $1, M_{\mathrm{ej}} / m_{2} \sim\left(\rho_{\mathrm{gr}} v^{2} / P_{1}\right) / \phi_{1}$ for Eq. $(2)$, so that $Q_{\mathrm{D}}^{\star}=$ $\left(P_{1} / 2 \rho_{\mathrm{gr}}\right) \phi_{1}$ gives a better approximation for $\phi_{1} \ll 1$. In other words, if $\phi_{1}$ is significantly smaller than 1 (i.e., for soft dust), Eq. (9) overestimates $Q_{\mathrm{D}}^{\star}$ by a factor of $\phi_{1}$. Thus, if we calculate shattering by adopting Eq. (9), grains are shattered less compared with Jones et al.'s case for soft dust. In this paper, because it is easy to interpret Eq. (9), we simply adopt it for KT10's formulation.

2.4.3 Size distribution of shattered fragments The power index of the size distribution of shattered fragments, $\alpha_{\mathrm{f}}$, may be reflected in the grain size distribution after shattering. The canonical value adopted in H10 is $\alpha_{\mathrm{f}}=3.3$, which is based on Jones et al. (1996). Here we also examine the cases of $\alpha_{\mathrm{f}}=2.3$ and 4.3 (Models $F$ and $\mathrm{G}$, respectively), considering a wide range obtained in experiments (Takasawa et al., 2011).

2.4.4 Grain velocities Grain motions are mainly driven by gyroresonance in the diffuse medium (Yan et al., 2004). Figure 1 presents the grain velocities as a function of grain radius in the WIM (Section 2.2). Large grains with $a \gtrsim 10^{-5} \mathrm{~cm}$ are accelerated to $\sim 20 \mathrm{~km} \mathrm{~s}^{-1}$ (i.e., similar velocities to the Alfvén velocity). Since larger grains 

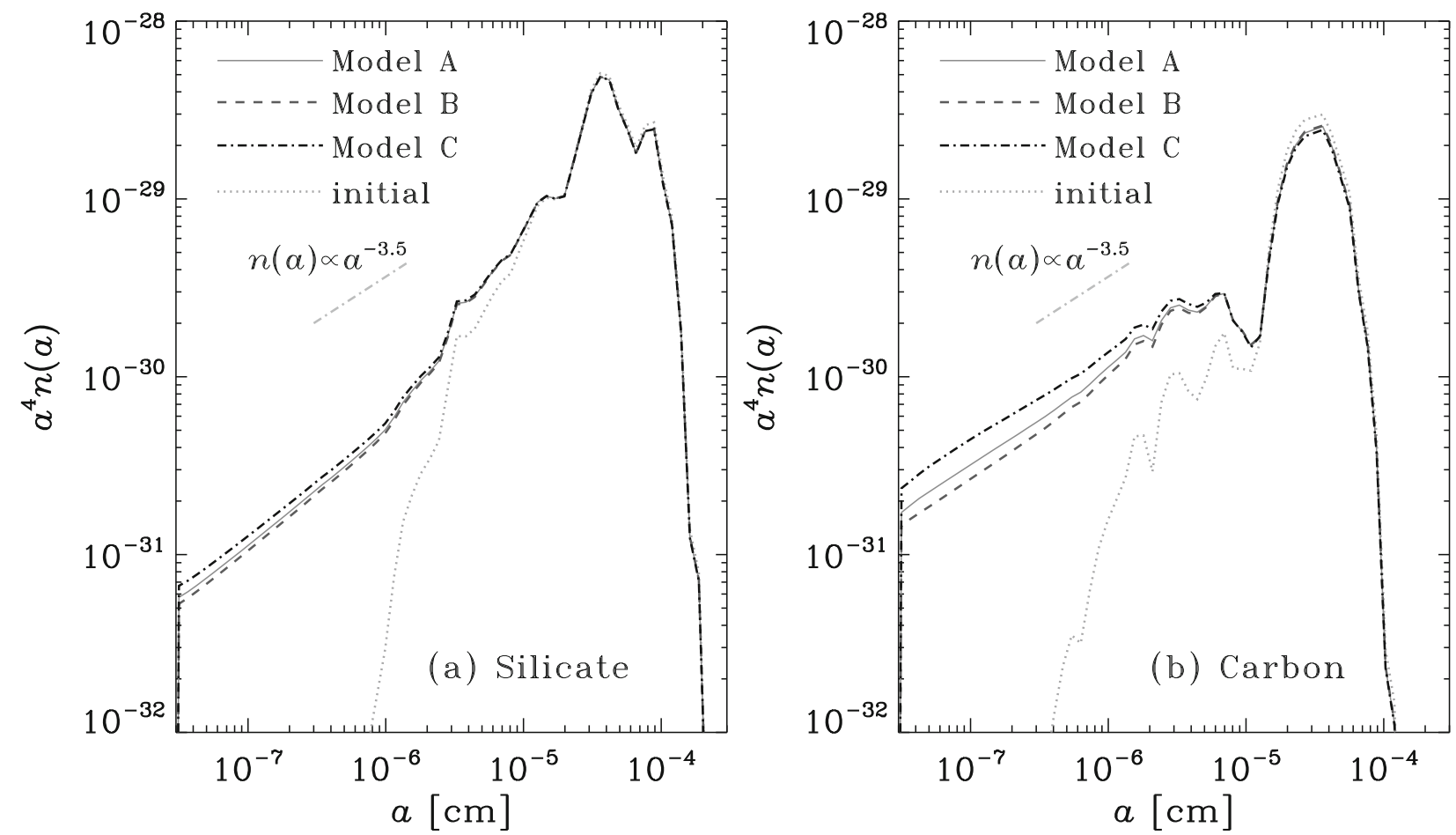

Fig. 2. Grain size distributions presented by multiplying $a^{4}$ to show the mass distribution in each logarithmic bin of the grain radius. The solid, dashed, and dot-dashed lines show the results at $t=10$ Myr for Models $\mathrm{A}, \mathrm{B}$, and $\mathrm{C}$ ( $f_{M}=0.4,0.3$, and 0.6 ), respectively. The dotted line presents the initial grain size distribution before shattering. Two grain species, (a) silicate and (b) carbonaceous dust, are shown. We also show a slope of 0.5 [i.e., $\left.n(a) \propto a^{-3.5}\right]$.

are coupled with larger-scale turbulence, the motions of larger grains are less affected by the damping by dissipation, which occurs on small scales. The dissipation scale depends on the physical condition of the ISM, especially on the ionization degree. Thus, the critical grain size, above which the grains are efficiently accelerated by gyroresonance, varies by the physical condition of the ISM and the power spectrum of the turbulence. Because of such a variation of the critical grain size, it is worth investigating how the shattered grain size distribution changes as a result of the variation of the critical grain size.

To make clear the dependence of the critical grain size denoted as $a_{\mathrm{c}}$, we also examine a model in which the size dependence of the grain velocity dispersion is simplified as

$$
v(a)=\left\{\begin{array}{c}
v_{\text {gyro }} \text { if } a \geq a_{\mathrm{c}} \\
0 \text { if } a<a_{\mathrm{c}}
\end{array}\right.
$$

An example with $a_{\mathrm{c}}=10^{-6} \mathrm{~cm}$ is shown in Fig. 1. We fix $v_{\text {gyro }}=20 \mathrm{~km} \mathrm{~s}^{-1}$. As long as $v_{\text {gyro }}>v_{\text {sh }}$, the variation of $v_{\text {gyro }}$ changes the timescale of shattering, which is roughly scaled with $v_{\text {gyro. }}^{-1}$. We examine $a_{\mathrm{c}}=10^{-5}$ and $10^{-6} \mathrm{~cm}$. This treatment of velocities is labeled as Model $\mathrm{H}$.

\section{Results}

\subsection{Ejected fraction of shocked material}

We examine the effect of $f_{M}$ on the grain size distribution. In Fig. 2, we show the grain size distributions at $t=10$ Myr for Models A, B, and C. In this paper, the grain size distributions are presented by multiplying $a^{4}$ to show the mass distribution in each logarithmic bin of the grain radius. We observe that the production of small grains is the most efficient for the largest $f_{M}$. This is simply because a larger fraction of the shocked material is ejected as fragments. However, the abundance of the smallest grains is not simply proportional to $f_{M}$, since the catastrophic disruption does not depend on $f_{M}$. The maximum ratio of $n(a)$ between $f_{M}=0.3$ and 0.6 is 1.3 for silicate and 1.6 for carbonaceous dust.

\subsection{Critical pressure}

We examine the dependence on the critical pressure $\left(P_{1}\right)$. The adopted values for the fiducial case (Model A) is shown in Table 2, while smaller and larger values for $P_{1}$ are also examined (Models D and E, respectively). In Fig. 3, we compare Models A, D, and $\mathrm{E}$ at $t=10 \mathrm{Myr}$. We find that the effect of $P_{1}$ is significant. Carbonaceous dust in Model D has the lowest $P_{1}$, so that the largest grains are efficiently shattered into smaller sizes. The mass loss rate of large grains with mass $m$ is proportional to $P_{1}^{-8 / 9}$ (Subsection 2.2); hence a low $P_{1}$ increases the number density of small $\left(a \lesssim 10^{-6} \mathrm{~cm}\right)$ grains. On the other hand, collisional cascades determine the slope of the grain size distribution as -3.5 , independent of $P_{1}$ (Hellyer, 1970; Tanaka et al., 1996; KT10). The slope is therefore given by -3.5 for $a \lesssim 10^{-6} \mathrm{~cm}$, a value $(-3.5)$ consistent with one derived observationally for the dust grains in the Milky Way and the Magellanic Clouds (Mathis et al., 1977; Pei, 1992).

To show the dependence on the treatment of total cratering volume, we also show the results calculated by the simple formulation of KT10 (see Subsection 2.4.2 for the formulation) in Fig. 3. We observe that KT10's formulation is in fair agreement with our formulation based on Jones et al. (1996) for the fiducial (Model A) and hard dust (Model 

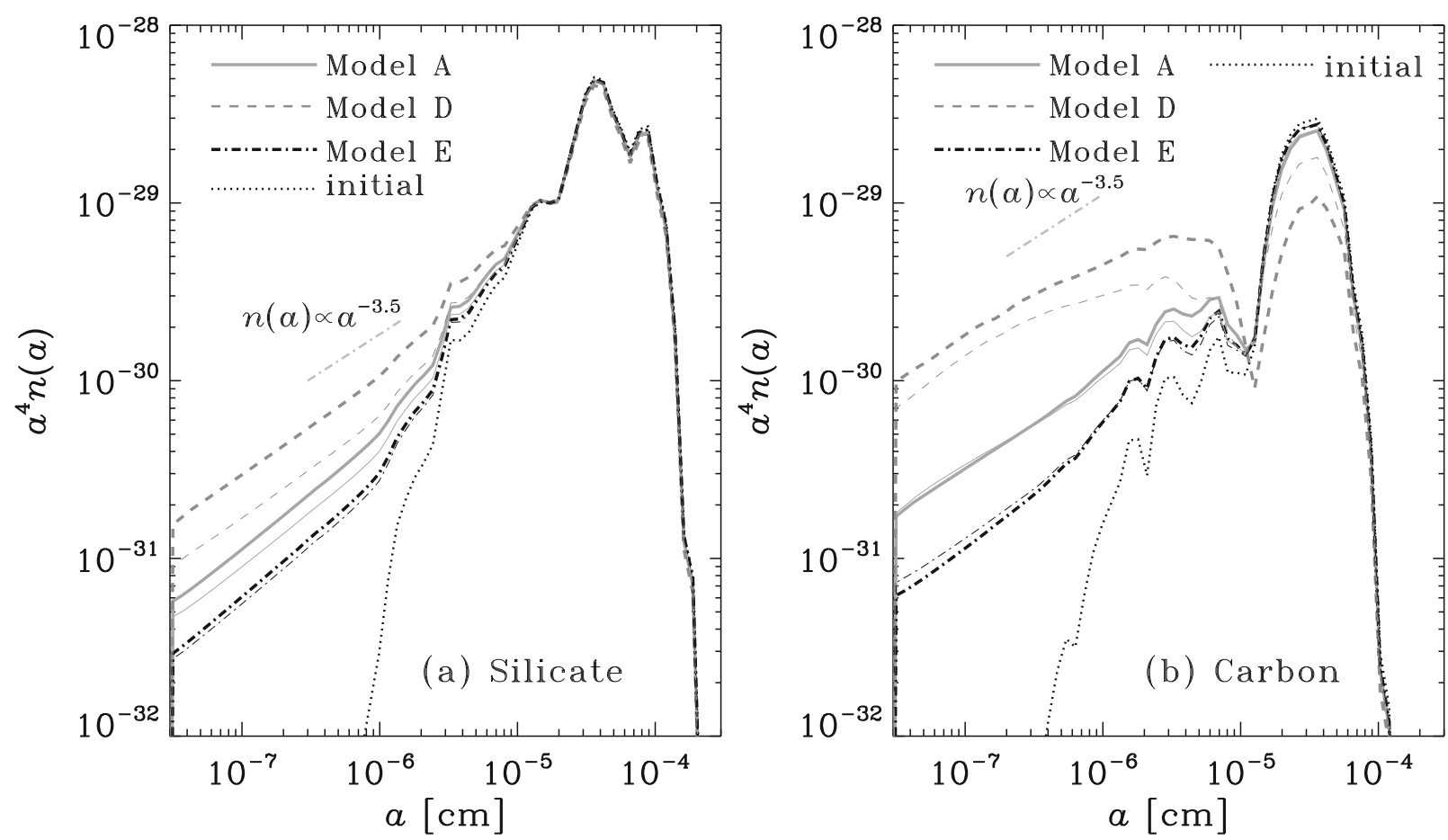

Fig. 3. Same as Fig. 2 but for Models A, D, and E. The thick solid, dashed, and dot-dashed lines show the results at $t=10$ Myr for Models A, D, and $\mathrm{E}$ (fiducial, hard, and soft grains), respectively, while the thin lines adopt the treatment of fragments by KT10 (the same line species shows the same model). The dotted line presents the initial grain size distribution before shattering. Two grain species, (a) silicate and (b) carbonaceous dust, are shown.

E), but the discrepancy is relatively large for the soft dust (Model D) because Eq. (9) is not a good approximation for the soft dust (see Subsection 2.4.2 for detailed discussions). The maximum ratio between the two formulations in Model $\mathrm{D}$ is 1.8 for silicate and 2.2 for carbonaceous dust. For carbonaceous dust, the maximum difference occurs around the complicated feature around $a \sim 10^{-5} \mathrm{~cm}$, but if we focus on the small grain production at $a<10^{-6} \mathrm{~cm}$, the ratio is 1.4. Thus, the uncertainty caused by the different formulations is just comparable to that caused by the difference in $f_{M}$. If we adopt the standard values for $P_{1}$ (i.e., Model A), it is concluded that the small grain production by shattering is described by the simple formulation by KT10 as precisely as more complicated framework of Jones et al. (1996).

\subsection{Size distribution of shattered fragments}

As we mentioned in Subsection 2.4.3, we assume that the size distribution of shattered fragments can be described by a power law function with power index $\alpha_{\mathrm{f}}$. In Fig. 4, we show the results for different values of $\alpha_{\mathrm{f}}$ (Models A, F, and G). We observe that the difference in $\alpha_{\mathrm{f}}$ affects the resulting grain size distributions. However, we also see that the difference between $\alpha_{\mathrm{f}}=3.3$ and 2.3 (Models A and F) is relatively small especially for silicate at $a>10^{-6} \mathrm{~cm}$, which implies that the grain size distribution does not necessarily becomes $n(a) \propto a^{-\alpha_{\mathrm{f}}}$. The grain size distribution in Model $\mathrm{G}$ at $a>10^{-6} \mathrm{~cm}$ is near to the initial grain size distribution, since the shattered grains are predominantly distributed at the smallest $\left(a \ll 10^{-6} \mathrm{~cm}\right)$ sizes.

In order to examine more clearly how the grain size distribution evolves depending on $\alpha_{\mathrm{f}}$, we show the results of Models $\mathrm{F}$ and $\mathrm{G}$ for $t=10,20,40$, and $80 \mathrm{Myr}$ in Fig. 5. The dip around $a \sim 2 \times 10^{-7} \mathrm{~cm}$, which is clearly seen at $t=80 \mathrm{Myr}$, is because of the velocity exceeding the shattering threshold around this grain radius (Fig. 1). This non-monotonic behavior of the velocity is due to the complexity of the grain charge as a function of grain size (Yan et al., 2004).

There is a qualitative difference in the evolution of grain size distribution between $\alpha_{\mathrm{f}}=2.3$ and 4.3. For $\alpha_{\mathrm{f}}=2.3$, the slope of the grain size distribution at $a \lesssim 10^{-6} \mathrm{~cm}$ changes, while for $\alpha_{\mathrm{f}}=4.3$, it evolves with a constant slope $\left[n(a) \propto a^{-\alpha_{\mathrm{f}}}\right]$ at small sizes. In the former case, the slope of the grain size distribution seems to approach $n(a) \propto a^{-3.5}$. The variation of slope occurs in a top-down manner; for example, for carbonaceous dust, the grain size distribution is nearly $n(a) \propto a^{-3.5}$ between $a=10^{-6}$ and $10^{-5} \mathrm{~cm}$ at 40 Myr, while it approaches $n(a) \propto a^{-3.5}$ at smaller sizes later at $80 \mathrm{Myr}$. This top-down behavior is because of the nature of shattering, which disrupts large grains into a lot of small pieces. This trend is also seen for $\alpha_{\mathrm{f}}=3.3$. Generally, if $\alpha_{\mathrm{f}} \lesssim 3.5$, the slope of grain size distribution tends to approach the MRN value (-3.5). On the contrary, if $\alpha_{\mathrm{f}} \gtrsim 4$ (see also Subsection 4.2), the resulting grain size distribution is determined by the grain size distribution of shattered fragments. Indeed, if $\alpha_{\mathrm{f}}>4$, the smallest particles, which are too small to disrupt large grains around the peak (i.e., $a \gtrsim 10^{-5} \mathrm{~cm}$ ), have the dominant contribution to the total fragment mass. Moreover, we remove the grains reaching the smallest-size bin $\left(a=3 \times 10^{-8} \mathrm{~cm}\right)$, and these grains do not affect the subsequent evolution of grain size distribution. Thus, for $\alpha_{\mathrm{f}}>4$, the fragments just keep their grain size distribution and eventually lost in reaching the smallest grain size.

We note that continuous shattering for such a long time as 

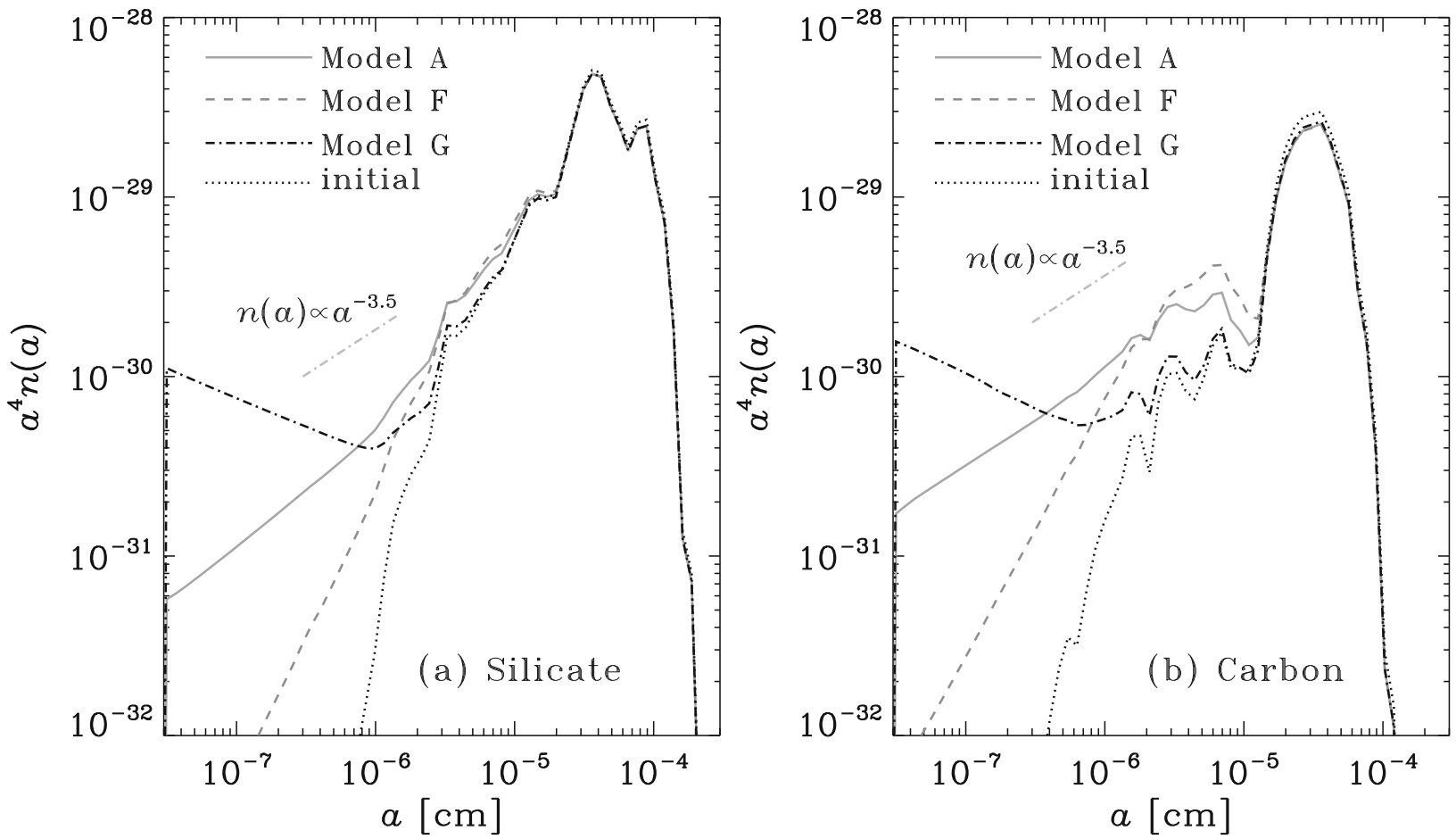

Fig. 4. Same as Fig. 2 but for Models A, F, and G, in order to investigate the effect of $\alpha_{\mathrm{f}}$ (power index of the grain size distribution of shattered fragments). The solid, dashed, and dot-dashed lines show the results at $t=10 \mathrm{Myr}$ for Models $\mathrm{A}, \mathrm{D}$, and $\mathrm{E}\left(\alpha_{\mathrm{f}}=3.3,2.3\right.$, and 4.3), respectively. The dotted line presents the initial grain size distribution before shattering.
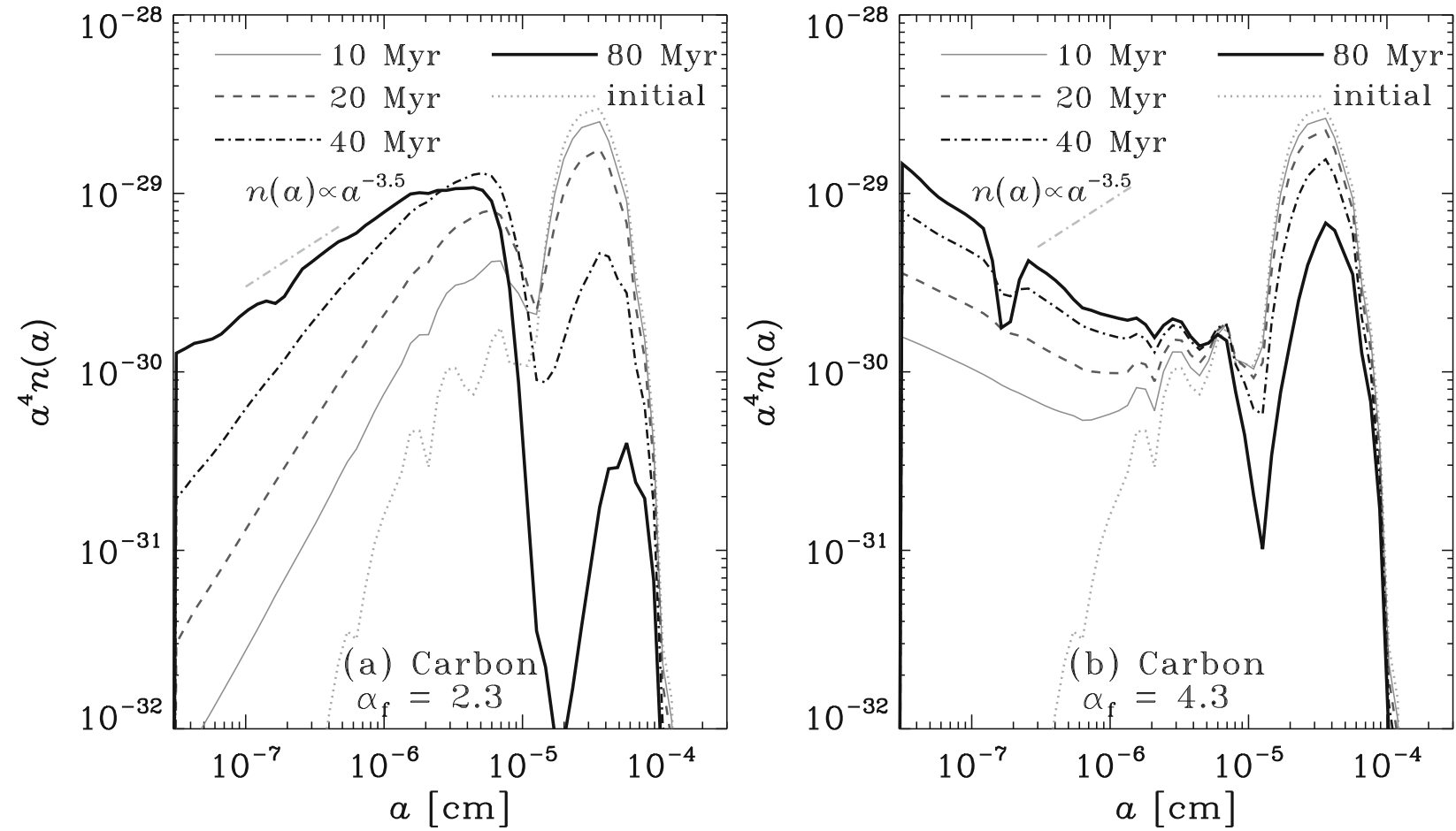

Fig. 5. Time evolution of grain size distribution for Models F and G in Panels (a) and (b), respectively, for carbonaceous dust. The thin solid, dashed, dot-dashed, and thick solid lines represent $t=10,20,40$, and $80 \mathrm{Myr}$, respectively. The dotted line presents the initial grain size distribution before shattering.

$80 \mathrm{Myr}$ is not applicable to the real WIM, since the lifetime of the WIM is probably shorter than 80 Myr. Yet, the above calculations for the long shattering durations show that, if grains are repeatedly shattered with $\alpha_{\mathrm{f}} \lesssim 3.5$, the final grain size distribution can approach a power law with an index of $\sim-3.5$. Therefore, shattering is a promising mechanism to produce an MRN-like grain size distribution. This issue is further discussed in Subsection 4.2. 

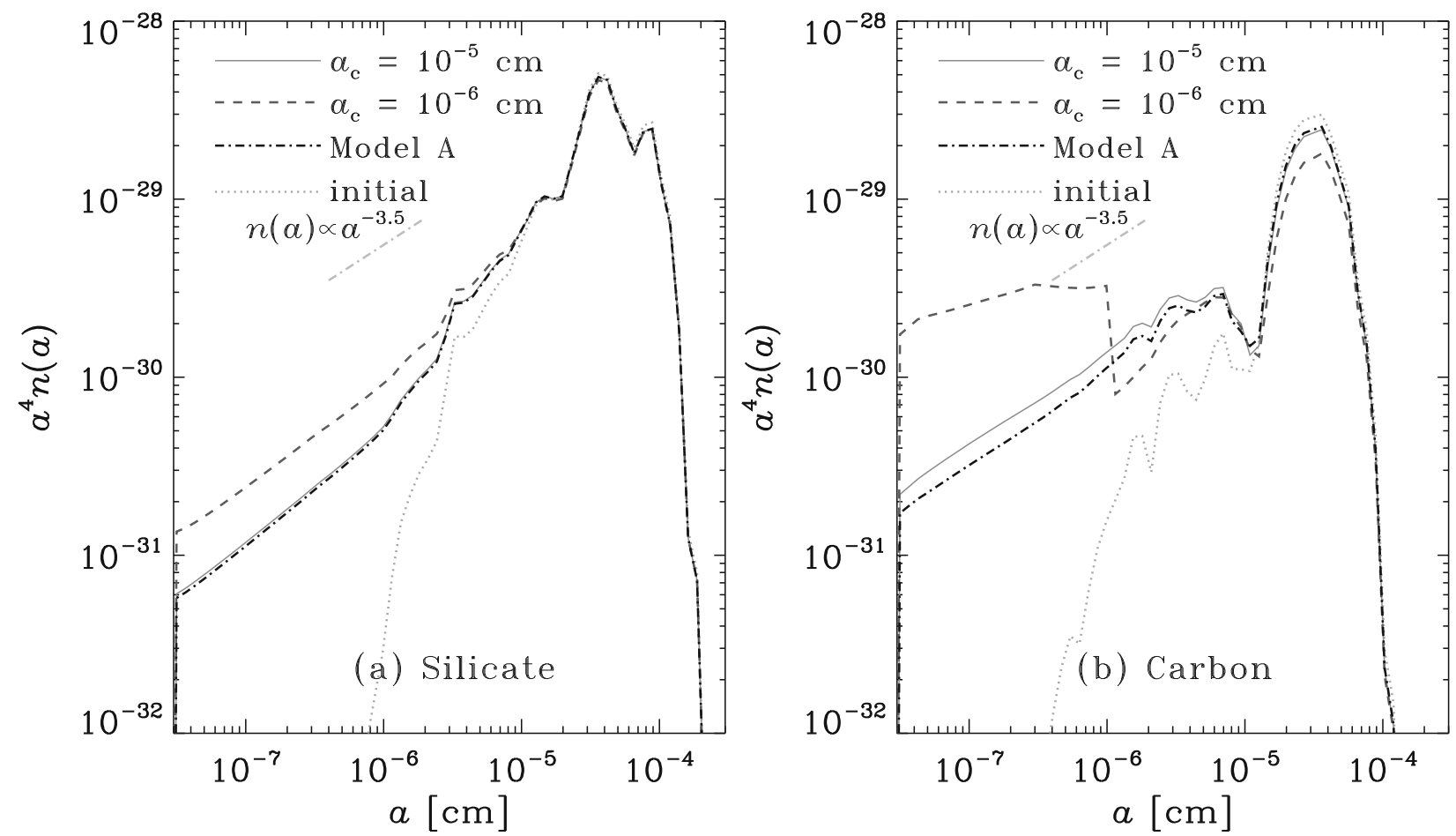

Fig. 6. Same as Fig. 2 but for Model $\mathrm{H}$ with various $a_{\mathrm{c}}$. The solid, and dashed lines show the results for $a_{\mathrm{c}}=10^{-5}$ and $10^{-6} \mathrm{~cm}$, respectively. The dotted line presents the initial grain size distribution before shattering, and the dot-dashed line shows the results for Model A as a reference.

\subsection{Grain velocities}

To understand the effect of grain velocities, we examine Model H, in which we use Eq. (10) and vary $a_{\mathrm{c}}$. In Fig. 6, we show the grain size distributions at $t=10 \mathrm{Myr}$ for $a_{\mathrm{c}}=10^{-5}$ and $10^{-6} \mathrm{~cm}$. We observe that the difference is clear at small grain sizes: because small grains are also shattered efficiently for small $a_{\mathrm{c}}$, the abundance of smallestsized grains is larger for smaller $a_{\mathrm{c}}$. Since carbonaceous dust is softer than silicate, the effect of $a_{\mathrm{c}}$ has a larger imprint on the grain size distribution of carbonaceous dust. Indeed, we clearly see the effect of $a_{\mathrm{c}}$ in the grain size distribution of carbonaceous dust in Fig. 6 (right panel). Thus, if small ( $a \lesssim 10^{-6} \mathrm{~cm}$ ) grains also acquire a larger velocity than the shattering threshold, it is predicted that the grain size distribution may not be approximated by a simple power law.

In Fig. 6, we also show the results of Model A as a reference. Since the grain velocities at $a<10^{-5} \mathrm{~cm}$ drops below $20 \mathrm{~km} \mathrm{~s}^{-1}$ for Model A (Fig. 6), Model H with $a_{\mathrm{c}}=$ $10^{-5} \mathrm{~cm}$ produce a more similar grain size distribution to Model A than Model H with $a_{\mathrm{c}}=10^{-6} \mathrm{~cm}$. Thus, the effect of $a_{\mathrm{c}}$ (i.e., the minimum size of grain size accelerated to the maximum velocity) is more important than the detailed functional form of $v(a)$ at $a<a_{\mathrm{c}}$.

\section{Discussion}

\subsection{Timescales}

In order to interpret the results above, it is useful to estimate a typical timescale of grain-grain collision. A grain with $a=a_{1}$ collide with a grain whose typical radius is $a_{2}$ with a frequency of

$$
\tau_{\text {coll }} \sim \frac{1}{\pi\left(a_{1}+a_{2}\right)^{2} n\left(a_{2}\right) \Delta a_{2} v_{12}},
$$

where $\Delta a_{2}$ is the radius interval of interest. Note that $n\left(a_{2}\right) \Delta a_{2}$ has a dimension of number density (Section 2). By using logarithmic size interval $\Delta \log a_{2}, \Delta a_{2} \sim$ $a_{2} \Delta \log a_{2}$. If we consider a $\log$ arithmic size bin so that $\Delta \log a_{2}$ is of order unity, we can replace $\Delta a_{2}$ with $a_{2}$. We estimate the typical timescale of small grain production by adopting the timescale on which a large grain is hit by a small grain $\left(a_{1} \gg a_{2}\right)$. In such a case, we obtain

$$
\begin{aligned}
\tau_{\text {coll }} \sim & \frac{1}{\pi a_{1}^{2} n\left(a_{2}\right) a_{2} v_{1}} \\
\sim & \frac{1}{\pi\left(\frac{a_{1}}{a_{2}}\right)^{2}\left[n\left(a_{2}\right) a_{2}^{4}\right] \frac{1}{a_{2}} v_{1}} . \\
\sim & 5.0\left(\frac{a_{2}}{a_{1}}\right)^{2}\left(\frac{a_{2}}{10^{-6} \mathrm{~cm}}\right)\left[\frac{n\left(a_{2}\right) a_{2}^{4}}{10^{-30}}\right]^{-1} \\
& \times\left(\frac{v_{1}}{20 \mathrm{~km} \mathrm{~s}^{-1}}\right)^{-1} \mathrm{Gyr} .
\end{aligned}
$$

Thus, we find that the timescale of small grain production becomes shorter if the typical size of colliding grains $\left(a_{2}\right)$ becomes small and the abundance of such grain $\left[n\left(a_{2}\right)\right]$ increases. For the initial grain size distribution, the grain radius is concentrated in a relatively narrow range around $\sim 3 \times 10^{-5} \mathrm{~cm}$ (Subsection 2.1; Fig. 2 and subsequent figures also show the initial grain size distribution), so that $a_{2} / a_{1} \sim 1 / 10$ and $a_{2} \sim 3 \times 10^{-6} \mathrm{~cm}$. Under such a condition, $\tau_{\text {coll }} \sim 1.5 \times 10^{8} \mathrm{yr}$, which is long compared with the typical duration treated in this paper (10 Myr). Thus, the large grains are hardly affected by shattering which is consistent with the fact that the grain size distribution around $10^{-5} \mathrm{~cm}$ is intact at $10 \mathrm{Myr}$ (Fig. 2). However, 

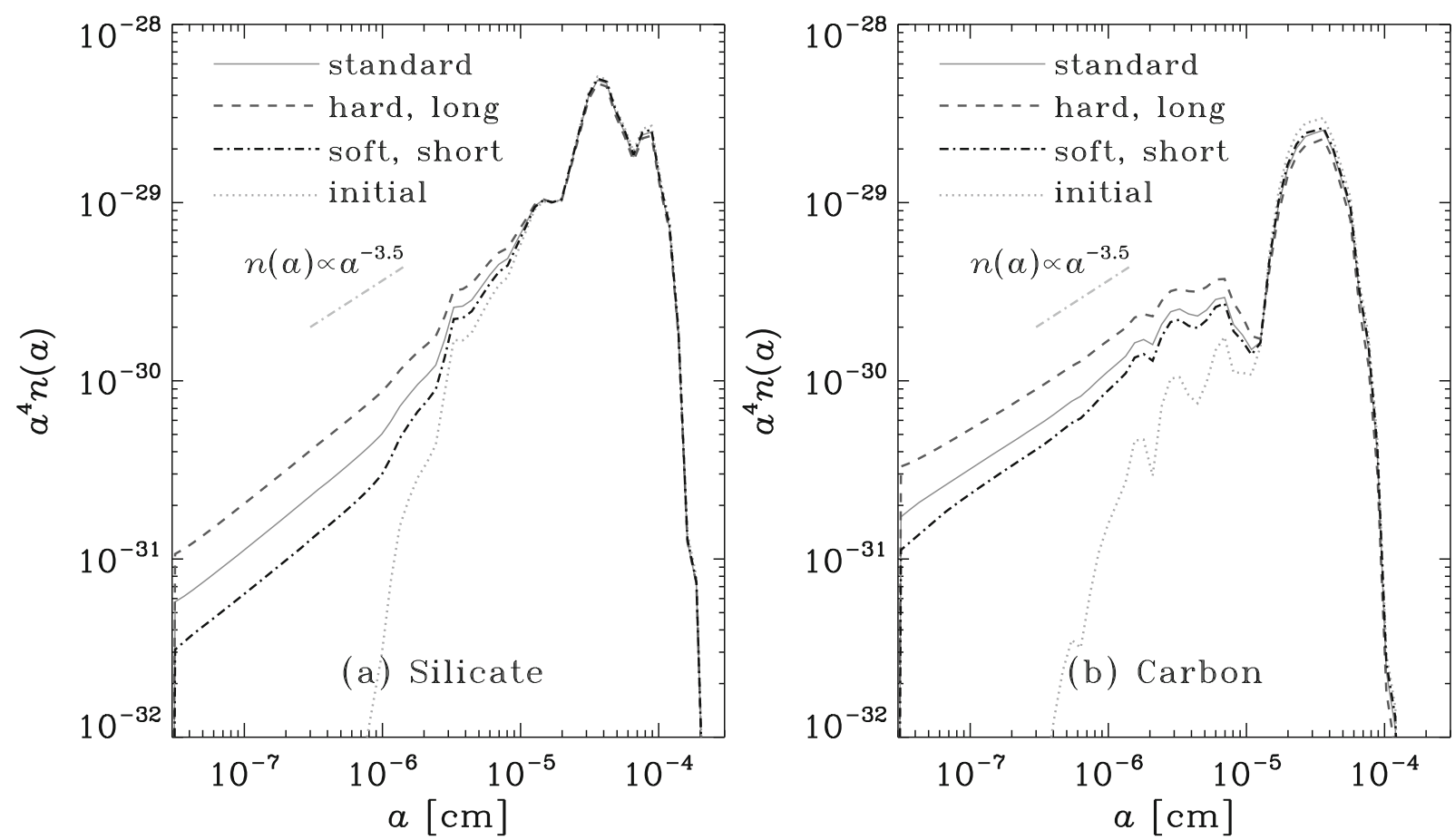

Fig. 7. Same as Fig. 3 but for constant $P_{1} / t$. The solid, dashed, and dot-dashed lines show the results for $\left(\right.$ a) $\left(P_{1}, t\right)=\left(3 \times 10^{11} \mathrm{dyn} \mathrm{cm}{ }^{-1}\right.$, $\left.10 \mathrm{Myr}\right)$, $\left(1 \times 10^{11} \mathrm{dyn} \mathrm{cm}^{-1}, 3.3 \mathrm{Myr}\right)$, and $\left(9 \times 10^{11} \mathrm{dyn} \mathrm{cm}^{-1}, 30 \mathrm{Myr}\right)$, respectively, for silicate, and $(\mathrm{b})\left(P_{1}, t\right)=\left(4 \times 10^{10} \mathrm{dyn} \mathrm{cm}^{-1}, 10 \mathrm{Myr}\right)$, $\left(1.3 \times 10^{10} \mathrm{dyn} \mathrm{cm}^{-1}, 3.3 \mathrm{Myr}\right)$, and $\left(1.2 \times 10^{11} \mathrm{dyn} \mathrm{cm}^{-1}, 30 \mathrm{Myr}\right)$, respectively, for carbonaceous dust. The dotted line presents the initial grain size distribution before shattering.

after a small amount of shattering, a lot of small grains are produced, and if grains with $a \lesssim 10^{-6} \mathrm{~cm}$ achieve $n(a) a^{4} \sim 10^{-30}$, the collision timescale with such small grains for a large grain $\left(a_{1} \sim 3 \times 10^{-5} \mathrm{~cm}\right)$ becomes an order of $10 \mathrm{Myr}$.

The timescale on which a large grain is lost because of repeated collisions of small grains can be estimated by

$$
\tau_{\mathrm{disr}} \sim\left(m_{1} / M_{\mathrm{ej}}\right) \tau_{\mathrm{coll}}
$$

This timescale is called disruption timescale. Here we show that the timescale of the loss of large $\left(a \sim 3 \times 10^{-5} \mathrm{~cm}\right)$ grains is consistent with the disruption timescale. For analytical convenience, we adopt KT10's formulation, which is roughly equivalent with Jones et al. (1996)'s formulation through Eq. (9). Since we are interested in the disruption of large grains by collisions with small grains, $m_{1} \gg m_{2}$ holds. Thus, from Eqs. (6) and (9), we obtain $\varphi \sim\left(a_{2} / a_{1}\right)^{3}\left(\rho_{\mathrm{gr}} v^{2} / P_{1}\right)$. If we adopt $a_{1} \sim 3 \times 10^{-5} \mathrm{~cm}$, $a_{2} \sim 3 \times 10^{-6} \mathrm{~cm}$, and $v \sim 20 \mathrm{~km} \mathrm{~s}^{-1}$, we obtain $\varphi \sim 0.22$ for carbonaceous grains. Thus, $M_{\mathrm{ej}} / m_{1} \sim 0.18$ from Eq. (8), and we finally obtain $\tau_{\text {disr }} \sim 5.5 \tau_{\text {coll }}$. Recalling that $\tau_{\text {coll }}$ is of an order of $10 \mathrm{Myr}$, the disruption timescale for the large grains is a few tens of Myr. This timescale is consistent with the loss of the grains around $a \sim 3 \times 10^{-5} \mathrm{~cm}$ in a few tens of Myr.

\subsection{Sensitivity to the parameters}

The resulting grain size distribution is sensitive to various parameters in different ways. As expected, shattering is more efficient for a smaller value of $P_{1}$ simply because the grains are softer. Since the cratering volume is approximately proportional to $1 / P_{1}$ (Jones et al., 1996), it is expected that similar grain size distributions are realized if we keep $P_{1} / t$ constant. To examine if this is the case, we compare the resulting grain size distributions for constant $P_{1} / t$ in Fig. 7. We examine $\left(P_{1}, t\right)=\left(3 \times 10^{11} \mathrm{dyn} \mathrm{cm}^{-1}, 10 \mathrm{Myr}\right),(1 \times$ $10^{11} \mathrm{dyn}^{-1}$, $3.3 \mathrm{Myr}$ ) (i.e., soft dust and short shattering duration), and $\left(9 \times 10^{11} \mathrm{dyn}^{-1}, 30 \mathrm{Myr}\right)$ (i.e., hard dust and long shattering duration) for silicate, and $\left(P_{1}, t\right)=(4 \times$ $\left.10^{10} \mathrm{dyn} \mathrm{cm}^{-1}, 10 \mathrm{Myr}\right),\left(1.3 \times 10^{10} \mathrm{dyn} \mathrm{cm}^{-1}, 3.3 \mathrm{Myr}\right)$, and $\left(1.2 \times 10^{11} \mathrm{dyn} \mathrm{cm}^{-1}, 30 \mathrm{Myr}\right)$ for carbonaceous dust. We observe that similar grain size distributions are indeed obtained within a factor of 3 . The difference arises because the scaling of the cratering volume (or $M_{\mathrm{ej}}$ ) with $P_{1}^{-1}$ is not perfect (Subsection 2.2).

The power index of fragment size distribution, $\alpha_{\mathrm{f}}$, is simply reflected in the resulting grain size distribution for $\alpha_{\mathrm{f}}=4.3$. However, if $\alpha_{\mathrm{f}}$ is smaller than 3.5 (e.g., the case with $\alpha_{\mathrm{f}}=2.3$ ), the slope of grain size distribution at $a \lesssim 10^{-5} \mathrm{~cm}$ continuously "steepens" and approaches to the slope consistent with MRN $\left[n(a) \propto a^{-3.5}\right]$ (Fig. 4). According to the analytical studies of shattering by Hellyer (1970), the power index of grain size distribution after shattering has a steady state value of $\simeq-3.5$ if the size distribution of shattered fragments is assumed to be a power law with an index smaller than 4 (i.e., $\alpha_{\mathrm{f}}<4$ ) (see also Tanaka et al., 1996), although we should note that the relative velocity between grains is a complicated function of grain sizes in our case. For $\alpha_{\mathrm{f}}>4$, since the mass occupied by the smallest fragments is dominating, the size distribution after shattering is just dominated by the smallest fragments, keeping a power law index of $-\alpha_{\mathrm{f}}$ at small sizes.

In general, larger grains have larger velocity dispersions 
if they are accelerated by turbulence, primarily because they are coupled with larger-scale turbulent motion which has larger velocity dispersions. Thus, even if the initial grain size distribution is biased to large grains with $a \gtrsim 10^{-5}$ $\mathrm{cm}$, these grains are efficiently shattered to produce a large abundance of small grains. If these shattered grains have also larger velocities than shattering threshold, they collide with each other, accelerating the production of small grains. Thus, as shown in Fig. 6, the effect of $a_{\mathrm{c}}$ (or the critical grain radius above which the grains have larger velocities than the shattering threshold) can be seen in the grain size distribution, causing a deviation from a monotonic power-law-like functional form. The imprint of $a_{\mathrm{c}}$ is more clear in carbonaceous dust than in silicate dust, since the former species is softer. Interestingly, Weingartner and Draine (2001) have shown that grain size distributions derived from the Milky Way extinction curves are complicated for carbonaceous dust, which may be explained by imprints of $a_{\mathrm{c}}$.

\subsection{Implication for the evolution of grain size distribu- tion in galaxies}

Inoue (2011) shows theoretically that the overall dust mass in metal-enriched systems such as the Milky Way is regulated by the balance between dust growth in molecular clouds and dust destruction by sputtering in SN shocks. While both these two mechanisms deplete small grains (Hirashita and Nozawa, 2013), shattering is a unique mechanism that reproduces the small grains. Indeed, Hirashita and Nozawa (2013) show that, unless we consider shattering, we underproduce the small grain abundance in the Milky Way (see also Asano et al., 2013b). As shown in Subsection 3.3, shattering not only produces small grains but also has an inherent mechanism of steepening the grain size distribution; that is, even if the grain size distribution of shattered fragments has $\alpha_{\mathrm{f}}$ smaller than 3.5, the resulting grain size distribution has a steeper dependence, approaching a slope consistent with the one derived by MRN [i.e., $\left.n(a) \propto a^{-3.5}\right]$. Thus, the robust prediction is that, if shattering is the dominant mechanism of small grain production, the grain size distribution approaches an MRN-like grain size distribution as long as $\alpha_{\mathrm{f}} \lesssim 3.5$. And if shattering is the dominant mechanism of small grain production, $\alpha_{\mathrm{f}}>4$ is rejected, because the grain size distribution in the Milky Way ISM has a power index of -3.5 (MRN).

In this paper, we have not included dust production by AGB stars. Even if the dust supply from AGB stars has a significant contribution, the results in this paper are not altered as long as the dust grains formed by AGB stars are biased to large $(\gtrsim 0.1 \mu \mathrm{m})$ sizes. Rather, the importance of shattering is emphasized because the additional dust production by AGB stars enhances the dust abundance, making grain-grain collision more frequent. Production of large grains from AGB stars is indicated observationally (Groenewegen, 1997; Gauger et al., 1998; Norris et al., 2012) and theoretically (Höfner, 2008; Mattsson and Höfner, 2011). We also neglected grain growth in molecular clouds, which is suggested to be a major dust formation mechanism even at high redshift (Mattsson, 2011; Valiante et al., 2011). Grain growth, however, cannot be a supplying mechanism of small grains.

For the shattering duration, since it is degenerate with the critical pressure of grains (hardness of grains), it is difficult to constrain it directly from the models. If grains experience shattering for several tens of Myr, the grain size distribution possibly approaches a power law whose power index is consistent with the MRN, if $\alpha_{\mathrm{f}} \lesssim 3.5$ (Fig. 5). Since the timescale of ISM phase exchange is comparable or shorter than that required for the grain size distribution to approach the MRN size distribution (O'Donnell and Mathis, 1997), shattering may occur intermittently. Even in this case, it is expected that grains are relaxed into an MRN-like simple power law if the total shattering duration reaches several tens of Myr. Such a duration for shattering is probable since it is much shorter than the grain lifetime $\left(\sim\right.$ a few $\times 10^{8}$ Myr; Jones et al., 1996).

\section{Conclusion}

Shattering is a viable mechanism of small grain production in the ISM both in nearby galaxies and in high-redshift galaxies. We have examined if grain size distributions predicted by shattering models are robust against the change of various parameters and formulations. Because of the uncertainty in $f_{M}$ (the fraction of the shocked material that is eventually ejected as fragments), the predicted grain size distribution after shattering is uncertain by a factor of 1.3 (1.6) for silicate (carbonaceous dust). We have identified $P_{1}$ (the critical pressure above which the original lattice structure is destroyed) as the most important quantity in determining the timescale of small grain production, and confirmed that the same $P_{1} / t$ ( $t$ is the duration of shattering) gives roughly the same grain size distributions after shattering within a factor of 3 .

A simpler and more intuitive formulation by KT10 is in good agreement with our model based on Jones et al. (1996) within a factor of 1.8 (1.4) for silicate (carbonaceous dust) if we focus on the small grain production at $a \lesssim 10^{-6} \mathrm{~cm}$. This is as small as the uncertainty caused by $f_{M}$. Thus, as long as we have an uncertainty in $f_{M}$, it is sufficient to adopt the simpler formulation by KT10. The size distribution of shattered fragments have minor effects as long as $\alpha_{\mathrm{f}} \lesssim 3.5$, since the grain size distribution is continuously steepened by shattering and become consistent with the MRN grain size distribution regardless of the value of $\alpha_{\mathrm{f}}(\lesssim 3.5)$.

The effect of the grain velocities as a function of grain radius can be seen more clearly in carbonaceous grains than in silicate, since the former species is shattered more easily. Thus, it is predicted that carbonaceous species has more complicated grain size distribution than silicate; in other words, the grain size distribution of carbonaceous dust can show some imprints of the grain velocity as a function of grain radius.

Acknowledgments. We are grateful to anonymous referees for helpful comments. HH has been supported through NSC grant 992112-M-001-006-MY3. HK gratefully acknowledges the support from Grants-in-Aid from MEXT (23103005).

\section{References}

Asano, R. S., T. T. Takeuchi, H. Hirashita, and A. K. Inoue, Dust formation history of galaxies: A critical role of metallicity for the dust mass growth by accreting materials in the interstellar medium, Earth Planets Space, 65, 213-222, 2013a.. 
Asano, R. S., T. T. Takeuchi, H. Hirashita, and T. Nozawa, What determines the grain size distribution in galaxies?, Mon. Not. R. Astron. Soc., 432, 637-652, 2013b.

Bianchi, S. and R. Schneider, Dust formation and survival in supernova ejecta, Mon. Not. R. Astron. Soc., 378, 973-982, 2007.

Bishop, J. E. L. and T. M. Searle, Power-law asymptotic mass distributions for systems of accreting or fragmenting bodies, Mon. Not. R. Astron. Soc., 203, 987-1009, 1983.

Bressan, A., F. Fagotto, G. Bertelli, and C. Chiosi, Evolutionary sequences of stellar models with new radiative opacities $\mathrm{II}-Z=0.02$, Astron. Astrophys. Suppl., 100, 647-664, 1993.

Désert, F.-X., F. Boulanger, and J. L. Puget, Interstellar dust models for extinction and emission, Astron. Astrophys., 237, 215-236, 1990.

Draine, B. T., Grain destruction in interstellar shock waves, Astrophys. Space Sci., 233, 111-123, 1995.

Draine, B. T., Interstellar dust grains, Ann. Rev. Astron. Astrophys., 41, 241-289, 2003.

Draine, B. T. and A. Li, Infrared emission from interstellar dust. I. Stochastic heating of small grains, Astrophys. J., 551, 807-824, 2001.

Dwek, E., The evolution of the elemental abundances in the gas and dust phases of the galaxy, Astrophys. J., 501, 643-665, 1998.

Gauger, A., Y. Y. Balega, P. Irrgang, R. Osterbart, and G. Weigelt, Highresolution speckle masking interferometry and radiative transfer modeling of the oxygen-rich AGB star AFGL 2290, Astron. Astrophys., 346, 505-519, 1998.

Groenewegen, M. A. T., IRC +10216 revisited. I. The circumstellar dust shell, Astron. Astrophys., 317, 503-520, 1997.

Guillet, V., G. Pineau des Forêts, and A. P. Jones, Shocks in dense clouds. III. Dust processing and feedback effects in C-type shocks, Astron. Astrophys., 497, 145-153, 2009.

Hellyer, B., The fragmentation of the asteroids, Mon. Not. R. Astron. Soc., 148, 383-390, 1970.

Hirashita, H. and T. Nozawa, Synthesized grain size distribution in the interstellar medium, Earth Planets Space, 65, 183-192, 2013.

Hirashita, H. and H. Yan, Shattering and coagulation of dust grains in interstellar turbulence, Mon. Not. R. Astron. Soc., 394, 1061-1074, 2009.

Hirashita, H., T. Nozawa, T. Kozasa, T. T. Ishii, and T. T. Takeuchi, Extinction curves expected in young galaxies, Mon. Not. R. Astron. Soc., 357, 1077-1087, 2005

Hirashita, H., T. Nozawa, H. Yan, and T. Kozasa, Effects of grain shattering by turbulence on extinction curves in starburst galaxies, Mon. Not. R. Astron. Soc., 404, 1437-1448, 2010 (H10).

Höfner, S., Winds of M-type AGB stars driven by micron-sized grains, Astron. Astrophys., 491, L1-L4, 2008.

Holsapple, K. A., Point source solutions and coupling parameters in cratering mechanics, J. Geophys. Res., 92, 6350-6376, 1987.

Holsapple, K. A., The scaling of impact processes in planetary sciences, Ann. Rev. Earth Planet. Sci., 21, 333-373, 1993.

Hoyle, F. and N. C. Wickramasinghe, Interstellar grains, Nature, 223, 450462, 1969.

Inoue, A. K., The origin of dust in galaxies revisited: The mechanism determining dust content, Earth Planets Space, 63, 1027-1039, 2011.

Inoue, A. K., H. Hirashita, and H. Kamaya, Conversion law of infrared luminosity of star-formation rate for galaxies, Publ. Astron. Soc. Jpn., 52, 539-543, 2000.

Jones, A., A. G. G. M. Tielens, D. Hollenbach, and C. F. McKee, Grain destruction in shocks in the interstellar medium, Astrophys. J., 433, 797$810,1994$.

Jones, A., A. G. G. M. Tielens, and D. Hollenbach, Grain shattering in shocks: The interstellar grain size distribution, Astrophys. J., 469, 740764, 1996.

Kawara, K. et al., Supernova dust for the extinction law in a young infrared galaxy at $z \sim 1$, Mon. Not. R. Astron. Soc., 412, 1070-1080, 2011.

Kim, S.-H., P. G. Martin, and P. D. Hendry, The size distribution of interstellar dust particles as determined from extinction, Astrophys. J., 422, 164-175, 1994.

Kobayashi, H. and H. Tanaka, Fragmentation model dependence of collision cascades, Icarus, 206, 735-746, 2010 (KT10).
Lodders, K., Solar system abundances and condensation temperatures of the elements, Astrophys. J., 591, 1220-1247, 2003.

Mathis, J. S., W. Rumpl, and K. H. Nordsieck, The size distribution of interstellar grains, Astrophys. J., 217, 425-433, 1977 (MRN).

Mattsson, L., Dust in the early Universe: Evidence for non-stellar dust production or observational errors?, Mon. Not. R. Astron. Soc., 414, 781-791, 2011.

Mattsson, L. and S. Höfner, Dust-driven mass loss from carbon stars as a function of stellar parameters. II. Effects of grain size on wind properties, Astron. Astrophys., 533, A42, 2011.

McKee, C. F., Dust destruction in the interstellar medium, in Interstellar Dust, Proc. of IAU 135, edited by L. J. Allamandola and A. G. G. M. Tielens, 431-443, Kluwer, Dordrecht, 1989.

Mizutani, H., Y. Takagi, and S. Kawakami, New scaling laws on impact fragmentation, Icarus, 87, 307-326, 1990.

Nakamura, A. and A. Fujiwara, Velocity distribution of fragments formed in a simulated collisional disruption, Icarus, 92, 132-146, 1991.

Nakamura, A. M., A. Fujiwara, and T. Kadono, Velocity of finer fragments from impact, Planet. Space Sci., 42, 1043-1052, 1994.

Norris, B. R. M., P. G. Tuthill, M. J. Ireland, S. Lacour, A. A. Zijlstra, F Lykou, T. M. Evans, P. Stewart, and T. R. Bedding, A close halo of large transparent grains around extreme red giant stars, Nature, 484, 220-222, 2012

Nozawa, T., T. Kozasa, and A. Habe, Dust destruction in the high-velocity shocks driven by supernovae in the early universe, Astrophys. J., 648 , 435-451, 2006.

Nozawa, T., T. Kozasa, A. Habe, E. Dwek, H. Umeda, N. Tominaga, K. Maeda, and K. Nomoto, Evolution of dust in primordial supernova remnants: Can dust grains formed in the ejecta survive and be injected into the early interstellar medium?, Astrophys. J., 666, 955-966, 2007 (N07).

O'Donnell, J. E. and J. S. Mathis, Dust grain size distributions and the abundance of refractory elements in the diffuse interstellar medium, Astrophys. J., 479, 806-817, 1997.

Ormel, C. W., D. Paszun, C. Dominik, and A. G. G. M. Tielens, Dust coagulation and fragmentation in molecular clouds. I. How collisions between dust aggregates alter the dust size distribution, Astron. Astrophys. 502, 845-869, 2009.

Pei, Y. C., Interstellar dust from the Milky Way to the Magellanic Clouds, Astrophys. J., 395, 130-139, 1992.

Takagi, Y., H. Mizutani, and S. Kawakami, Impact fragmentation experiments of basalts and pyrophyllites, Icarus, 59, 462-477, 1984.

Takasawa, S. et al., Silicate dust size distribution from hypervelocity collisions: Implications for dust production in debris disks, Astrophys. $J$., 733, L39, 2011.

Tanaka, H., S. Inaba, and K. Nakazawa, Steady-state size distribution for the self-similar collision cascade, Icarus, 123, 450-455, 1996.

Umeda, H. and K. Nomoto, Nucleosynthesis of zinc and iron peak elements in population III type II supernovae: Comparison with abundances of very metal poor halo stars, Astrophys. J., 565, 385-404, 2002.

Valiante, R., R. Schneider, S. Salvadori, and S. Bianchi, The origin of dust in high-redshift quasars: the case of SDSS J1148+5251, Mon. Not. R. Astron. Soc., 416, 1916-1935, 2011.

Weingartner, J. C. and B. T. Draine, Dust grain-size distributions and extinction in the Milky Way, large magellanic cloud, and small magellanic cloud, Astrophys. J., 548, 296-309, 2001.

Yamasawa, D., A. Habe, T. Kozasa, T. Nozawa, H. Hirashita, H. Umeda, and K. Nomoto, The role of dust in the early universe. I. Protogalaxy evolution, Astrophys. J., 735, 44, 2011.

Yan, H., A. Lazarian, and B. T. Draine, Dust dynamics in compressible magnetohydrodynamic turbulence, Astrophys. J., 616, 895-911, 2004.

Zhukovska, S., H.-P. Gail, and M. Trieloff, Evolution of interstellar dust and stardust in the solar neighbourhood, Astron. Astrophys., 479, 453480, 2008.

H. Hirashita (e-mail: hirashita@asiaa.sinica.edu.tw) and H. Kobayashi 